\title{
Improving Short-Term Memory Performance of Healthy Young Males Using Alpha Band Neurofeedback
}

\author{
Barış Gökşin, Bülent Yılmaz*, and Kutay İçöz \\ School of Engineering, Abdullah Gül University, Kayseri, Turkey
}

\begin{abstract}
To examine whether it was possible to improve short-term memory performance of healthy participants by increasing relative alpha band power $(7-11.5 \mathrm{~Hz})$ using neurofeedback, we first converted a commercial EEG device (EmotivEpoc) to a neurofeedback tool and collected data from 11 healthy Turkish male graduate students in five neurofeedback sessions. Before and after neurofeedback training, a memorization task using 10 English words and their Turkish meanings was applied to all participants. The results indicated that 6 out of 11 participants were able to enhance their relative alpha band power with respect to other bands in the frequency spectrum during neurofeedback sessions. Although there was no obvious improvement in their short-term memory performance, we may conclude that neurofeedback training was beneficial for the participants to focus their minds consciously. However, it is not easy to mention that neurofeedback training certainly improved or was irrelevant with short-term memory performance. This study is important in the sense that for such a focused group the use of a commercial, customized low-cost EEG device was shown to be feasible for neurofeedback training sessions.
\end{abstract}

Keywords: short-term memory; neurofeedback; alpha band

Citation: Gökşin, B., Yılmaz, B., \& İçöz, K. (2019). Improving short-term memory performance of healthy young males using alpha band neurofeedback. NeuroRegulation, 6(1), 15-22. http://dx.doi.org/10.15540/nr.6.1.15

*Address correspondence to: Bülent Yılmaz, Department of Electrical \& Electronics Engineering, School of Engineering, Abdullah Gül University, Sümer Campus - 38080 Kayseri, Turkey. Email: bulent.yilmaz@agu.edu.tr

Copyright: (C) 2019. Gökşin et al. This is an Open Access article distributed under the terms of the Creative Commons Attribution License (CC-BY).

\section{Edited by:}

Rex L. Cannon, PhD, Knoxville Neurofeedback Group, Knoxville, Tennessee, USA

Reviewed by:

Rex L. Cannon, PhD, Knoxville Neurofeedback Group, Knoxville, Tennessee, USA

Randall Lyle, PhD, Mount Mercy University, Cedar Rapids, lowa, USA

\section{Introduction}

It is a fact that human physical and psychological functions start to degrade with aging, which causes a significant decrease in life quality (Wang \& Hsieh, 2013). Considering all the cognitive functions of the brain, memory is likely to be one of the most important cognitive abilities for individuals to sustain their life quality and productivity.

Klimesch, Doppelmayr, Pachinger \& Ripper (1997) reported that there was a relationship between memory performance and the alpha band activity. When an individual's resting-state alpha activity was high, long-term memory performance was also high (Klimesch, 1999). Besides that, it was reported that not only alpha activity is related with memory but also some other bands' activities are related to memory performance (Reis et al., 2016). Wang and Hsieh
(2013) reported that frontal-midline theta band enhancement improves working memory and attention. Vernon et al. (2003) reported that enhancement of sensorimotor rhythm (SMR; 12-15 $\mathrm{Hz}$ ) activity improves working memory performance. Although there are many reports that memory performance is associated with alpha activity, Bauer (1976) studied whether increasing $(8.5-12.5 \mathrm{~Hz})$ alpha activity improves recall performance, but he could not observe such a relationship for short-term memory.

Neurofeedback training (NFT) method gives subjects audio/visual feedback of their brain wave activity so that they can learn to control their own brain rhythm. In addition, it has been studied for several decades as an alternative to traditional medication for some psychological disorders such as attentiondeficit/hyperactivity disorder (ADHD; Friel, 2007), 
substance addiction (Sokhadze, Cannon, \& Trudeau, 2008), epilepsy (Sterman \& Egner, 2006), and autism (Coben, Linden, \& Myers, 2010). NFT has also been used to enhance the cognitive performance of healthy participants. Besides its function in psychological disorders, NFT has been shown to have some positive effects on cognitive performance of healthy individuals having different occupations, for example, musicians and surgeons (Gruzelier, Egner \& Vernon, 2006; Ros et al., 2009). Although there are many studies which support that NFT has positive effects on some areas including cognitive performance improvement using alpha band power (Marzbani, Marateb, \& Mansourian, 2016), there are limited studies which show the effects of NFT on short-term memory in the literature (Conway, Cowan, Bunting, Therriault \& Minkoff, 2002).

Lecomte and Juhel (2011) investigated whether increasing upper alpha band $(10-12 \mathrm{~Hz})$ power over theta band $(4-7 \mathrm{~Hz})$ power could improve short-term memory performance or not. In their electroencephalography (EEG) experiments, C3, C4, and $\mathrm{Cz}$ (ref) electrodes were used, and an audiovisual feedback was employed. After four neurofeedback training sessions, they showed that subjects could increase alpha band power and alpha/theta band power, however, there was no memory performance improvement in elderly participants. Kober et al. (2015) examined whether increasing the SMR $(12-15 \mathrm{~Hz})$ band power or upper alpha $(10-12 \mathrm{~Hz})$ band power could improve shortterm memory performance in post-stroke patients. In EEG experiments, $\mathrm{Cz}$ electrode was used for SMR, $\mathrm{Pz}$ electrode was used for upper-alpha training, and a visual feedback was employed. After 10 neurofeedback training sessions, they showed that participants who took the SMR training were able to improve their visuospatial short-term memory performance. Moreover, participants who took the upper-alpha training could improve their working memory performance. Nan et al. (2012) investigated the potential of increase in the individual alpha band power to improve the short-term memory performance. In the experiments, only $\mathrm{Cz}$ electrode was used, and a visual feedback was employed. After 20 neurofeedback training sessions, they showed that participants could increase their individual alpha band power and improve the shortterm memory performance. Finally, Wang (2017) examined whether increasing the alpha band power could improve the working memory performance in the students with ADHD. In EEG experiments, only $\mathrm{FCz}$ electrode was used. After 10 neurofeedback training sessions, he showed that the participants could increase alpha band power and improve working memory performance.

In this study, we first converted a wireless EEG device (Emotiv EPOC) to a neurofeedback tool. Later, we examined whether it was possible to improve shortterm memory performance of 11 healthy participants by increasing relative alpha band power using neurofeedback training. Before and after 5 days of neurofeedback training sessions, we measured the improvement on participants' short-term memory by performing a test that consisted of memorizing and recalling the Turkish meanings of some English words which were not previously known by the participants.

\section{Materials and Methods}

\section{Participants}

The 11 subjects who participated in the experiments were chosen among male graduate students, with an average age of 29 and with standard deviation of \pm 3.04 . One participant was left-handed and five of them wore glasses. For the experiment, there was no payment to the participants except for a chocolate bar for every session. Before experiments, written approval from Abdullah Gül University Internal Review Board and oral consent from participants were received.

\section{EEG Recordings}

During the experiments the setup shown in Figure 1 of the Emotiv EPOC system, a 14-channel wireless EEG device with $128-\mathrm{Hz}$ sampling frequency, was used. According to Emotiv manufacturer, the "measurement accuracy of the device is technically: minimum voltage resolution $0.51 \mu \mathrm{V}, 14$-bit accuracy, 0.2-43 Hz bandwidth" ("How Accurate Is Your Detection", 2019). The voltage resolution was suitable to measure EEG signals, because the EEG signal varied around a voltage level that was above $\pm 10 \mu \mathrm{V}$. The reference electrodes were placed on the left and right mastoids. Another important component of the EEG recoding was the real-time signal acquisition and processing of the brain waves. For this purpose we first installed MATLAB 2012b (32-bit version) to our computer and Visual $\mathrm{C}++2010$ to compile MATLAB. Later, we used the modified version of a MATLAB file called "eeglogger.m" that was developed and shared by the manufacturer of the wireless headset. By means of the modifications we were able to convert the system into a neurofeedback tool (necessary files can be found as supplementary materials). In this work we used only P8 electrode (according to the international 10-20 system), 
because it was one of the suitable places to record the signals without getting affected from the eyeblinks.

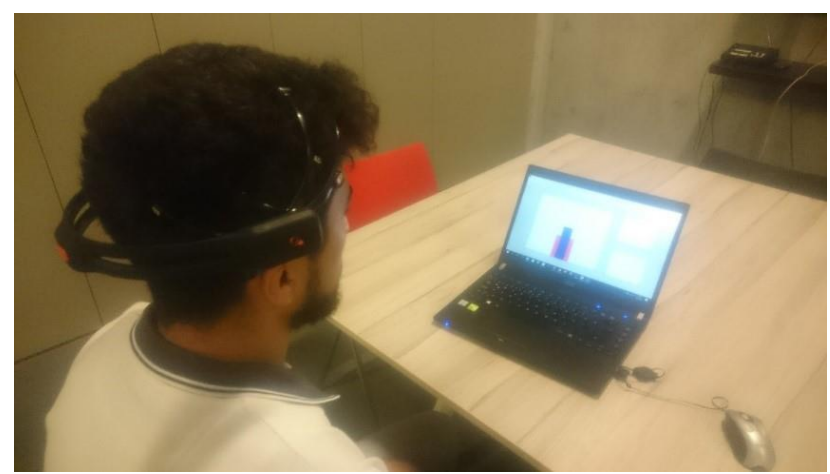

Figure 1. Experimental setup.

\section{Study Design}

Before the neurofeedback training sessions were started, a baseline EEG measurement obtained with eyes open and a pretraining test was given to the participants to be able to measure short-term memory performance of the participants. The pretraining test included 10 words such as exigency, innocuous, and desultory, which were not known by the participants beforehand. Firstly, the participants were let memorize the words by looking at the selected words shown one by one on the screen followed by its meaning. There was no time limit in this part. Once the memorization part was over, it was requested from each participant to remember the meanings when each English word was shown on the screen. No multiple-choice type approach was followed. Memorization time, recall time, and the number of accurately recalled words were recorded manually. After the pretraining test, five sessions of neurofeedback training were applied (Figure 2). Each day only one session was applied, and the training sessions lasted at most for 10 days according to the availability of the participants. One session included three subsessions and five trials constituted one subsession, and thus one training session included 15 trials. Each trial lasted for $45 \mathrm{~s}$, and $10 \mathrm{~s}$ were placed between two subsequent trials. After five trials (one subsession) the participants had enough time to rest, approximately one to two minutes. After the neurofeedback training sessions, a posttraining test was given to the participants. This test also included 10 different English words with similar memorization difficulty. The recall performances of the participants were manually recorded.

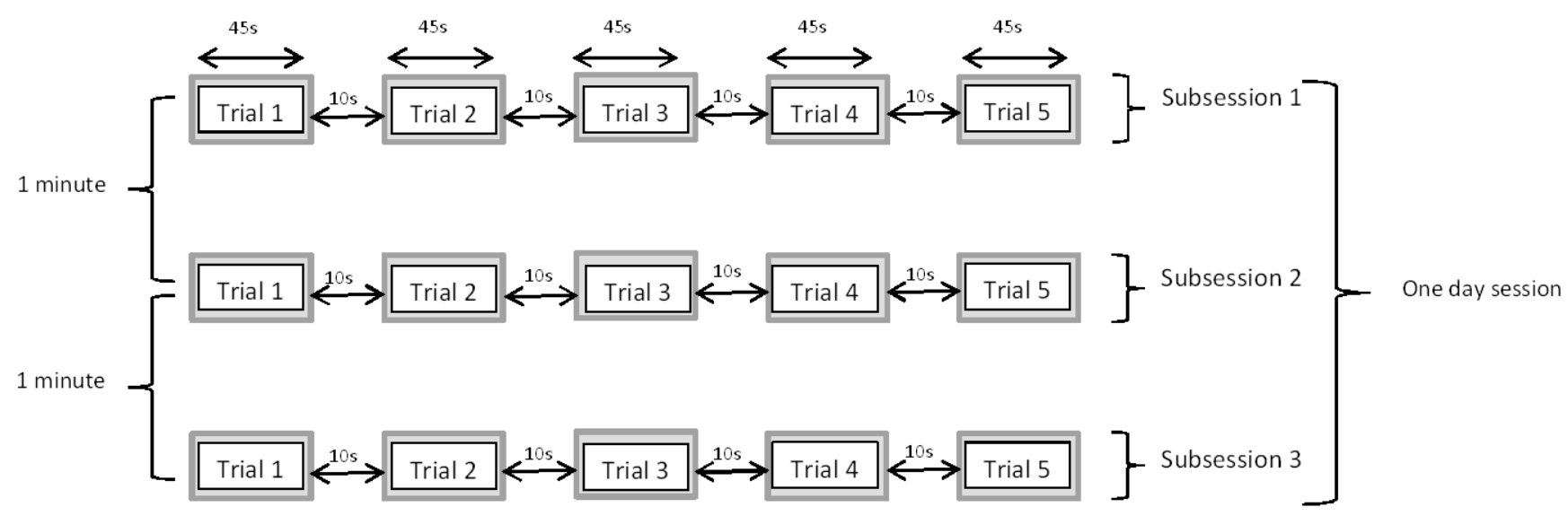

Figure 2. Neurofeedback training paradigm. 


\section{Neurofeedback Training}

During the design stage, we recorded EEG signals from an individual with closed and open eyes for a few times, and then found the intersection of these two cases. The data collected with open and closed eye method made it possible to observe alpha band synchronization and desynchronization, which can be used to figure out alpha frequency band by using band crossings (Nan et al., 2012). We found that open and closed eyes spectra intersect at approximately $7 \mathrm{~Hz}$ and $11.5 \mathrm{~Hz}$, and we decided to use this band for all participants in our experiments. Relative band power (RBP) was used as the neurofeedback parameter. RBP represents the ratio between the absolute band power in $7-11.5 \mathrm{~Hz}$ range and the absolute band power in $3.5-35 \mathrm{~Hz}$ range.

During the training, the participants were able to see the panels shown in Figure 3 . We oriented the participants about the system and the feedback panel before the sessions had started. The blue bar on the left panel indicated the dynamically changing relative alpha band power, and the red bars depicted the mean value of the relative alpha band power measured during the baseline recordings. The mean power values were updated after each training subsession.

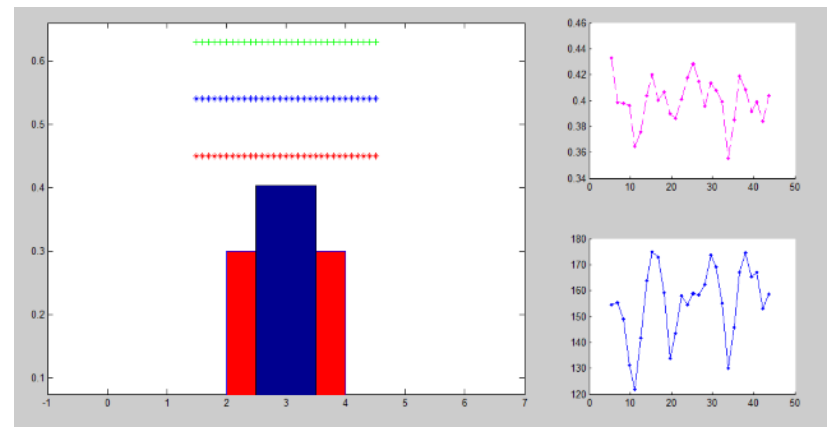

Figure 3. User interface of neurofeedback training sessions.

The participants tried to increase the blue bar's level to pass the red bars (baseline level), which worked as a visual feedback. We also used an audio feedback as a beep sound when the level was surpassed. Some participants asked us to turn the sound off, because they thought that it was distracting their concentration. On that panel the red, blue, and green lines showed some levels that the participant would aim to pass as a challenge. They were utilized to motivate the participants. On the right panel, the dashed pink line showed relative alpha band power, and the blue line indicated the absolute alpha band power. We asked them to focus on the left panel only.

Before the training session started, several thinking strategies were advised to the participants. For example, the alpha power generally is related to the relaxation process, and positive thinking such as thinking about the family, friends and natural scenes increase the alpha power (Nan et al., 2012). On the first, second, and third sessions it was requested from the participants to determine the best thinking strategy that helped them to increase their alpha power. However, on the fourth and fifth sessions, it was requested not to find any new thinking strategies but to focus on what they had figured out before to increase the relative alpha band power as much as they could. At the end of the subsessions, the participants were able to see their performances as shown in Figure 4.

\section{Real-time EEG Processing}

Before performing the real-time spectrum analysis of the EEG signals obtained, several preprocessing steps were applied. When we acquired the EEG signals, we observed that there was a positive offset around $4150 \mu \mathrm{V}$, and we removed that offset by subtracting 4150 from each value in the dataset. After offset removal, the signal was fed into a fifth-degree Butterworth high-pass filter whose cut-off frequency was $3 \mathrm{~Hz}$. Because we were only interested in the alpha band power, we selected this cut-off in order not to affect any spectral coefficients in that band. When we did not perform the filtering excessive lowfrequency power dominated the spectral coefficients.

In the power spectrum computations Welch's method was used. This method first divides data into windows (200 samples per iteration). Adjacent windows have some overlap $(50 \%)$ between each other, and the method calculates the spectrum of these windows. Finally, averaging is applied to compute the spectrum of the data. The spectrum coefficients were computed for every $0.25 \mathrm{~Hz}$ (frequency resolution). During the training sessions, before computing alpha band power values in realtime, sufficient number of samples had to be collected. The first computed power spectrum was seen on the screen once we acquired 700 samples. During the spectrum computations, the last 512 samples were used (last $4 \mathrm{~s}$ ). In addition, in real-time computations, there was no artifact removal. 

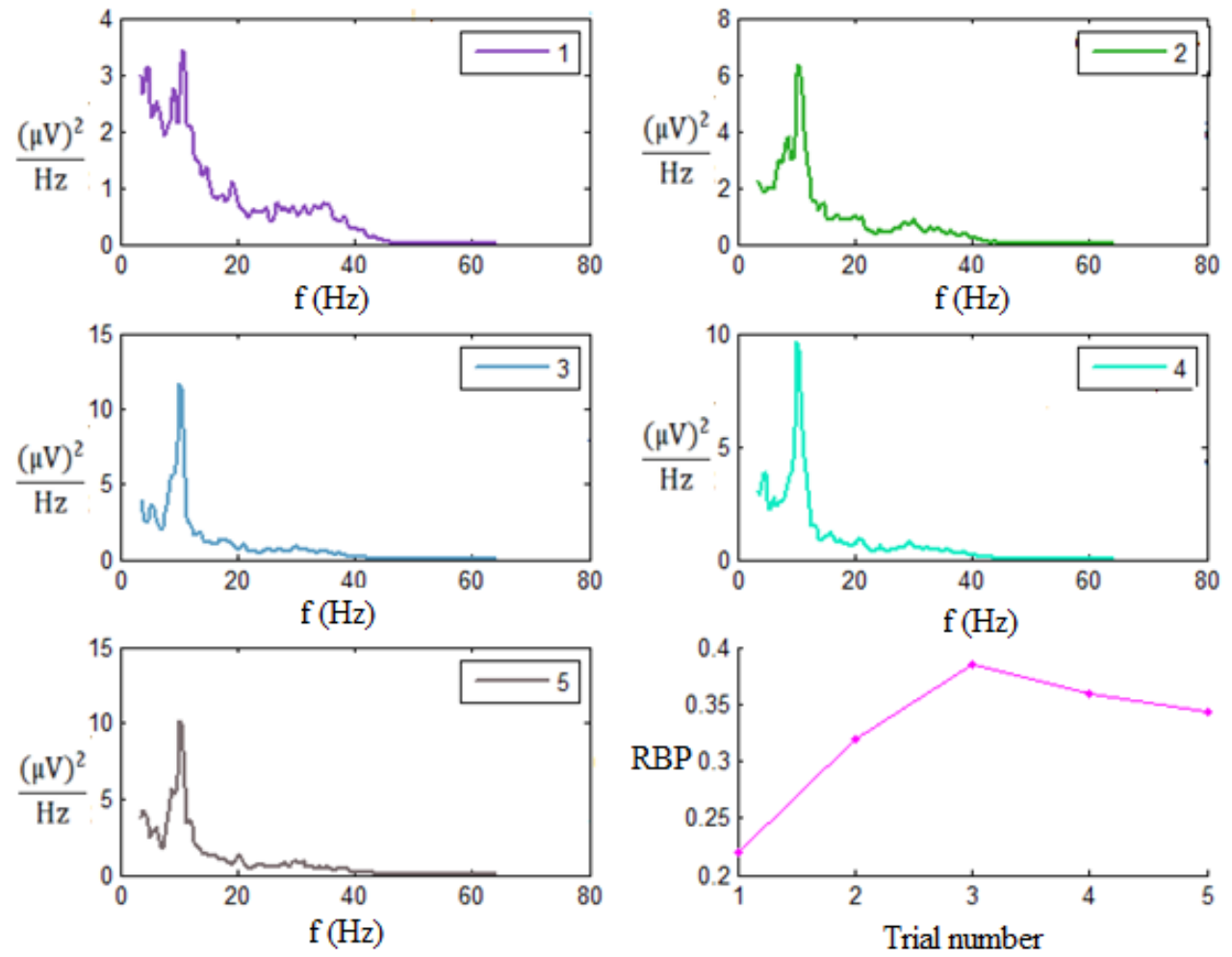

Figure 4. EEG spectra of the participant \#10 from the first day, third subsession. Relative band power (RBP) is also shown on the bottom-right panel.

\section{Results}

Table 1 summarizes the results obtained during this study indicating the average alpha band power (ARABP) values during the baseline recordings and each training session, pretraining and posttraining memorization and recall durations and the number of correct answers.

It is important to note that 6 out of 11 participants were able to increase their ARABP values between $2.7 \%$ and $11.75 \%, 3$ participants slightly increased their ARABP $\sim 1 \%$, and 2 of them slightly decreased their ARABP values below approximately $1 \%$. Taking all participants into consideration, it was observed that the ARABP increase was on average $4.4 \%$ as depicted in Figure 5. Including only the successful participants, who increased alpha band power over $1 \%$, they were able to increase the ARABP $7.73 \%$ as depicted in Figure 5.
Four participants who increased ARABP were also able to increase the number of the correct recalls, which they increased recall performances between 2 and 4 folds. One of the successful participants could not increase or decrease the number of correct recalls. One participant increased the ARABP (>1\%) but decreased the number of correct recalls. Two participants who were able to slightly increase ARABP could increase the number of correct recalls (5 to 7 folds). One participant who was able to slightly increase alpha band power decreased the number of correct recalls. Two participants decreased their alpha band power but increased the number of correct recalls ( 2 to 2.25 folds). When all participants were considered, they were able to increase the number of correct recalls 2.63 fold on average. All subjects increased posttraining memorization duration compared to the pretraining memorization duration of $4.76 \mathrm{~s}$ per word. 


\section{Table 1}

Results obtained during neurofeedback experiments combined in one table.

\begin{tabular}{|c|c|c|c|c|c|c|c|c|c|c|c|c|}
\hline Participant & Baseline & Day 1 & Day 2 & Day 3 & Day 4 & Day 5 & $\begin{array}{c}\text { Pretest } \\
\text { Memorization } \\
\text { Duration (s) }\end{array}$ & $\begin{array}{c}\text { Posttest } \\
\text { Memorization } \\
\text { Duration (s) }\end{array}$ & $\begin{array}{c}\text { Pretest } \\
\text { Recall } \\
\text { Duration } \\
\text { (s) }\end{array}$ & $\begin{array}{l}\text { Posttest } \\
\text { Recall } \\
\text { Duration } \\
\text { (s) }\end{array}$ & $\begin{array}{c}\text { Pretest } \\
\text { \# of } \\
\text { Correct } \\
\text { Answers }\end{array}$ & $\begin{array}{l}\text { Posttest } \\
\text { \# of } \\
\text { Correct } \\
\text { Answers } \\
\end{array}$ \\
\hline$\# 1$ & 0.1601 & 0.1978 & 0.1999 & 0.1841 & 0.2515 & 0.2284 & 95 & 123 & $\mathrm{~N} / \mathrm{A}$ & 59 & 3 & 9 \\
\hline \#2 & 0.1071 & 0.1491 & 0.1646 & 0.1937 & 0.1726 & 0.1885 & 86 & 91 & 120 & 136 & 3 & 2 \\
\hline \#3 & 0.2128 & 0.2055 & 0.2143 & 0.2241 & 0.1945 & 0.2382 & 46 & 75 & 69 & 61 & 1 & 7 \\
\hline$\# 4$ & 0.1759 & 0.2116 & 0.1892 & 0.2000 & 0.1950 & 0.2185 & 117 & 182 & 103 & 105 & 2 & 8 \\
\hline \#5 & 0.2158 & 0.2458 & 0.2201 & 0.2057 & 0.2486 & 0.1897 & 42 & 45 & 45 & 85 & 4 & 1 \\
\hline \#6 & 0.1567 & 0.1513 & 0.1622 & 0.1498 & 0.1641 & 0.1505 & 82 & 116 & 113 & 105 & 2 & 4 \\
\hline$\# 9$ & 0.1789 & 0.1887 & 0.1848 & 0.1833 & 0.1817 & 0.1860 & 64 & 101 & $\mathrm{~N} / \mathrm{A}$ & 86 & 1 & 5 \\
\hline$\# 10$ & 0.3039 & 0.2301 & 0.313 & 0.3071 & 0.3163 & 0.3233 & 88 & 153 & $\mathrm{~N} / \mathrm{A}$ & 62 & 4 & 9 \\
\hline$\# 11$ & 0.1073 & 0.1529 & 0.1394 & 0.1792 & 0.1926 & 0.1612 & 94 & 162 & 103 & 94 & 4 & 7 \\
\hline Mean & 0.1856 & 0.2048 & 0.2154 & 0.2217 & 0.2311 & 0.2295 & 82 & 129 & - & - & 3.27 & 6.18 \\
\hline
\end{tabular}
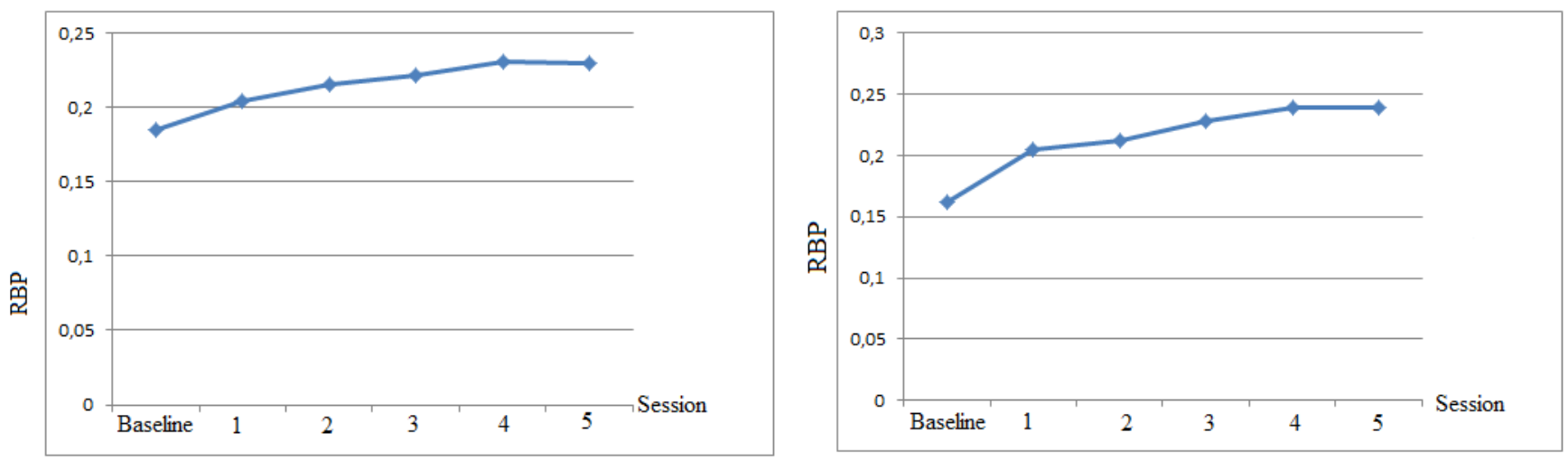

Figure 5. All (left) and successful (right) participants' average relative alpha band power enhancement day by day.

\section{Discussion}

In this study we asked two related questions about the relative alpha band power during the neurofeedback training sessions and short-term memory improvement. The first question was the following: "Is it possible to increase the alpha band power intentionally when compared to respect to other bands (theta, beta, and gamma bands) of the
EEG spectrum?" According to our results, the answer to this question may be positive. More than half of the participants were able to increase their alpha band power when compared to the other bands.

The second question we investigated was the following: "Were participants, who increased relative alpha band power, also able to improve short-term memory performance?" In this study 8 out of 11 participants were able to increase the number of 
words correctly remembered (correct recalls). However, for the successful participants, only four of them were able to increase the number of correct recalls. Therefore, we were not able to give a positive answer to this question. This did not mean that neurofeedback training was not related to short-term memory performance. These findings comply with the results of the study undertaken by Lecomte and Juhel (2011). In that study, a group of elderly participants experienced four neurofeedback training sessions, and the participants were requested to memorize a list of words. They found that the participants were able to increase alpha band power; however, there were not any improvements in the memory performance.

Feedback from the participants revealed that positive thinking such as thinking of the family, friends, beautiful scenes and so forth was effective in increasing the alpha band power. However, not only positive thinking increases alpha band power but also some participants used negative motives such as getting angry with someone or something in order to improve the desired band power. This finding complies with the study of Nan et al. (2012).

Another finding was that when some subjects focused on one type of thinking style, this increased their alpha band power first, but decreased later. In one participant, the relative alpha band power firstly increased, and then decreased after the third trial of a subsession. This observation was generally true especially after the third and fourth trials. This finding might have arisen due to the exhaustion of the brain while thinking about the same feeling or object for a long time. Therefore, it may be useful for someone to have a rest during the neurofeedback sessions by changing the thinking style or the work, which they did from one session to another. One neurological explanation of this suggestion might be that the activation of another neural network might help the exhausted one rest.

A limitation of this study was the number of subjects who participated in the neurofeedback sessions. Inclusion of only 11 graduate students in the study might have biased the interpretation of the results; however, we think that this study is important in the sense that for such a focused group the use of a commercial, customized low-cost EEG device was shown to be feasible. Another limitation was the number of sessions and their durations. Although there are studies reported in the literature which included experiments with five sessions such as Escolano, Aguilar, and Minguez (2011) and Zoefel,
Huster, and Herrmann (2011), the number of training sessions and session durations might not be sufficient for some participants to adapt with the environment, and determine strategies to control their brains in parallel with the aim of the study, which was increasing the alpha band power. However, we thought that if we increased the number of sessions or durations the participants would get bored, and this might affect the results negatively. In addition, several subjects mentioned that they got bored of viewing bar graphs in all training sessions. If we were to use a game in the neurofeedback sessions, it would have been more interesting and motivating for the participants, and they might have been more enthusiastic about the sessions. In our future endeavor we will increase the number of participants, the neurofeedback training sessions and their durations to investigate the phenomenon on the children with learning difficulties.

In the neurofeedback literature, during the experiments participants were requested to find their way to synchronize with the feedback signals (visual or auditory) by themselves. There was an "aha" point which participants could understand and synchronize with the screen or the sound (Collura, 2000). However, in our study, due to the limited number of sessions we advised some thinking strategies to the participants before the neurofeedback training. Participants indicated that they could not fully synchronize with the blue bar during training, which means they were not sure whether one type of thinking style increased or decreased the alpha power. However, at the end of the trial, they mentioned that a particular thinking style was generally useful in increasing the alpha power.

In the literature, this is the first study in which Turkish students were trained via neurofeedback sessions in order to improve their short-term memory performances by using English words.

According to the findings reported by Klimesch in 1997, memory retrieval performance of subjects who had high resting state peak of alpha frequency, which is the frequency of maximum amplitude in alpha band, was better than others. In a future study, memory performance improvement may be examined by increasing the peak alpha frequency with increased number of participants and number of sessions using motivating games. 


\section{Conclusion}

In conclusion, the findings of this study are neither sufficient to prove that neurofeedback training improves the short-term memory performance, nor it is irrelevant with the short-term memory performance. However, we may mention that the neurofeedback training is beneficial for the subjects to orient their conscious minds to their goals.

\section{Acknowledgment}

We would like to thank the graduate students at Abdullah Gül University, Kayseri, Turkey, who participated our neurofeedback training sessions and pre and post-training tests.

\section{Author Disclosure}

Authors have no grants, financial interests, or conflicts to disclose.

\section{References}

Bauer, R. H. (1976). Short-term memory: EEG alpha correlates and the effect of increased alpha. Behavioral Biology, 17(4), 42533. http://dx.doi.org/10.1016/S0091-6773(76)90793-8

Coben, R., Linden, M., \& Myers, T. E. (2010). Neurofeedback for autistic spectrum disorder: A review of the literature. Applied Psychophysiology and Biofeedback, 35(1), 83-105. http://dx.doi.org/10.1007/s10484-009-9117-y

Collura, T. F. (2000). Practical issues concerning EEG biofeedback devices, protocols and methods. Retrieved November 20, 2017 from http://openeeg.sourceforge.net/arch/att-0944/01 part

Conway, A. R. A., Cowan, N., Bunting, M. F., Therriault, D. J., \& Minkoff, S. R. B. (2002). A latent variable analysis of working memory capacity, short-term memory capacity, processing speed, and general fluid intelligence. Intelligence, 30(2), 163183. http://dx.doi.org/10.1016/S0160-2896(01)00096-4

Escolano, C., Aguilar, M., \& Minguez, J. (2011, August). EEGbased upper alpha neurofeedback training improves working memory performance. Paper presented at the 33rd Annual International Conference of the IEEE Engineering Medicine and Biology Society, Boston, MA. http://dx.doi.org/10.1109 /IEMBS.2011.6090651

Friel, P. N. (2007). EEG biofeedback in the treatment of attention deficit hyperactivity disorder. Alternative Medicine Review, 12(2), 146-151.

Gruzelier, J., Egner, T., \& Vernon, D. (2006). Validating the efficacy of neurofeedback for optimising performance. Progress in Brain Research, 159, 421-431. http://dx.doi.org/10.1016 /S0079-6123(06)59027-2

How Accurate Is Your Detection? (2019, January 11). Retrieved from https://emotiv.zendesk.com/hc/en-us/articles /204993629-How-accurate-is-your-detection-

Klimesch, W. (1997). EEG-alpha rhythms and memory processes. International Journal of Psychophysiology, 26(1-3), 319-340. http://dx.doi.org/10.1016/S0167-8760(97)00773-3

Klimesch, W. (1999). EEG alpha and theta oscillations reflect cognitive and memory performance: A review and analysis. Brain Research Reviews, 29(2-3), 169-195. http://dx.doi.org /10.1016/S0165-0173(98)00056-3
Klimesch, W., Doppelmayr, M., Pachinger, T., \& Ripper, B. (1997). Brain oscillations and human memory: EEG correlates in the upper alpha and theta bands. Neuroscience Letters, 238(1-2), 9-12. http://dx.doi.org/10.1016/s0304-3940(97)00771-4

Kober, S. E., Schweiger, D., Witte, M., Reichert, J. L., Grieshofer, P., Neuper, C., \& Wood, G. (2015). Specific effects of EEG based neurofeedback training on memory functions in poststroke victims. Journal of NeuroEngineering and Rehabilitation, 12, 107. http://dx.doi.org/10.1186/s12984-0150105-6

Lecomte, G., \& Juhel, J. (2011). The effects of neurofeedback training on memory performance in elderly subjects. Psychology, 2(8), 846-852. http://dx.doi.org/10.4236 /psych.2011.28129

Marzbani, H., Marateb, H. R., \& Mansourian, M. (2016). Neurofeedback: A comprehensive review on system design, methodology and clinical applications. Basic and Clinical Neuroscience, 7(2), 143-158. http://dx.doi.org/10.15412 /J.BCN.03070208

Nan, W., Rodrigues, J. P., Ma, J., Qu, X., Wan, F., Mak, P.-I., ... Rosa, A. (2012). Individual alpha neurofeedback training effect on short-term memory. International Journal of Psychophysiology, 86(1), 83-87. http://dx.doi.org/10.1016 /j.ijpsycho.2012.07.182

Reis, J., Portugal, A. M., Fernandes, L., Afonso, N., Pereira, M., Sousa N., \& Dias N. S. (2016). An alpha and theta intensive and short neurofeedback protocol for healthy aging workingmemory training. Frontiers in Aging Neuroscience, 8, 157. http://dx.doi.org/10.3389/fnagi.2016.00157

Ros, T., Moseley, M. J., Bloom, P. A., Benjamin, L., Parkinson, L. A., \& Gruzelier, J. H. (2009). Optimizing microsurgical skills with EEG neurofeedback. BMC Neuroscience, 10, 87. http://dx.doi.org/10.1186/1471-2202-10-87

Sokhadze, T. M., Cannon, R. L., \& Trudeau, D. L. (2008). EEG biofeedback as a treatment for substance use disorders: Review, rating of efficacy, and recommendations for further research. Applied Psychophysiology and Biofeedback, 33(1), 1-28. http://dx.doi.org/10.1007/s10484-007-9047-5

Sterman, M. B., \& Egner, T. (2006). Foundation and practice of neurofeedback for the treatment of epilepsy. Applied Psychophysiology and Biofeedback, 31(1), 21-35. http://dx.doi.org/10.1007/s10484-006-9002-x

Vernon, D., Egner, T., Cooper, N., Compton, T., Neilands, C., Sheri, A., \& Gruzelier, J. (2003). The effect of training distinct neurofeedback protocols on aspects of cognitive performance. International Journal of Psychophysiology, 47(1), 75-85. http://dx.doi.org/10.1016/S0167-8760(02)00091-0

Wang, J.-R., \& Hsieh, S. (2013). Neurofeedback training improves attention and working memory performance. Clinical Neurophysiology, 124(12), 2406-2420. http://dx.doi.org /10.1016/j.clinph.2013.05.020

Wang, Z. (2017). Neurofeedback training intervention for enhancing working memory function in attention deficit and hyperactivity disorder (ADHD) Chinese students. NeuroQuantology, 15(2), 277-283. http://dx.doi.org/10.14704 /nq.2017.15.2.1073

Zoefel, B., Huster, R. J., \& Herrmann, C. S. (2011). Neurofeedback training of the upper alpha frequency band in EEG improves cognitive performance. Neurolmage 54(2), 1427-1431. http://dx.doi.org/10.1016/j.neuroimage.2010.08.078

Received: January 25, 2019

Accepted: February 8, 2019

Published: March 25, 2019 